\title{
New Economy, Risikokapital und die Mythen des Internet*
}

In diesem ArtikeI wird aus einer neoinstitutionalistischen Perspektive die Funktionsweise risikokapitalfinanzierter Unternehmen analysiert. Als Beispiel dient dabei der Internet-Hype von 1995 bis 2000. Aufgrund des schnellen Wachstums der Internetunternehmen und der Möglichkeit, Anteile von sehr jungen Unternehmen an den Börsen zu handeln, war diese Branche ein geeignetes Investitionsfeld für Risikokapitalgesellschaften. Insofern davon ausgegangen wurde, dass eine schnelle Marktpenetration über Erfolg oder Misserfolg von Unternehmen im Internet entscheidet, waren nicht kurzfristige Profitabilität, sondern Nutzerzahlen, Bildschirmbesucher und Umsatzsteigerungen wichtig. Da die Geschäftsmodelle risikokapitalfinanzierter Unternehmen vorrangig auf einem Nachfluss von Geldern aus dem Kapitalmarkt aufgebaut sind, ist es für sie von wesentlicher Bedeutung, die „Meilensteine“ auch zu erreichen, die sie bezüglich Nutzerzahlen, Umsatzsteigerung und Ergebnisverbesserung aufgestellt haben. Kapitalmarktorientierte Unternehmen nutzten deswegen ausgeprägt Möglichkeiten, um Nutzerzahlen, Bildschirmbesucher, Umsatz und Unternehmensergebnisse zu schönen. Die so entstehenden „Unternehmensrealitäten" basieren darauf, dass die Geldnachflüsse aus dem Kapitalmarkt sich fortsetzen. Wenn sie ausbleiben, brechen die Unternehmen wie Kartenhäuser in sich zusammen.

\section{Einleitung}

Der Begriff der New Economy wurde häufig als ein Sammelbegriff für Firmen der Branchen E-Commerce, Telekommunikation und Biotechnologie genutzt. Er stand aber auch für die ,neue Wirtschaftsform der aufkommenden Wissensgesellschaft", die „Wissen, Information und Kreativität" als "Treibstoff" für neues Wachstum ansah (vgl. Froitzheim 2001: 23; siehe auch Feng et al. 2001: 3). In diesem Artikel geht es nicht darum, die New Economy über diffuse Merkmale wie ,informationstechnisch basiert", "global" und "vernetzt" (vgl. z.B. Castells 2000) zu bestimmen, sondern ihre Ausbildung vor dem Hintergrund eines institutionalisierten Risikokapitalmarktes zu erklären. Risikokapital ermöglicht Unternehmensfinanzierungen in Bereichen, in denen keine anderen Finanzierungsquellen vorhanden sind. Einer Unternehmerin oder einem Unternehmer mit einer Produkt- oder Dienstleistungsidee, einer neu- en Technologie oder einer originellen Anwendungsidee für eine existierende Technik stehen als Financiers häufig nur Risikokapitalgeber zur Verfügung. Nicht zuletzt durch das Verbot des Wuchers ist es Banken nicht immer möglich, Kredite an junge Unternehmen auszugeben. Aufgrund der fehlenden Sicherheit, des hohen Ausfallrisikos und des großen Prüfaufwandes müsste eine Bank sehr hohe Zinsen verlangen. Deshalb finanzieren Banken Jungunternehmer, wenn überhaupt, nur bis zu einer Summe, für die diese mit Sach-oder Grundeigentum bürgen können.

Mit Business Angels, die Unternehmen in der frühen Gründungsphase finanzieren, mit Risikokapitalgesellschaften und Anlegern an Börsen für Wachstumsunternehmen wie der NASDAQ haben sich Institutionen ausgebildet, die in die Marktnische der Finanzierung von innovativen Wachstumsunternehmen vorgestoßen sind. Diese Art der Finanzierung ermöglicht bei hohem Risiko nicht nur jährliche Renditen von 20 Prozent und mehr, son-

* Ich danke Norbert Huchler, Marianne Schröder, Alexander Schulze-Fielitz, Eric Treske, Peter Walgenbach und den Gutachtern des Berliner Journals für Soziologie für hilfreiche Kritik an früheren Fassungen des Artikels. 
dern wird auch von den Medien, der Politik und den Wirtschaftsverbänden als eine wichtige Voraussetzung der Innovationsfähigkeit einer Volkswirtschaft gepriesen. So wird die frühe Entwicklung von Mikrocomputern, Personal-Computern, Festplatten, Diskettenlaufwerken und Computer-Workstations sowie von genetisch hergestellten Medikamenten in den USA häufig auf die Existenz einer institutionalisierten Risikofinanzierung zurückgeführt (vgl. z.B. Southwick 2001). Im Gegenzug wurde das relative Zurückbleiben Deutschlands, Englands oder Frankreichs bei der Entwicklung hoch innovativer Produkte lange Zeit auf das Fehlen eines funktionierenden Risikokapitalmarkts zurückgeführt. Die Etablierung einer aktiven Risikokapitalszene und die Gründung von Börsen für Wachstumsunternehmen am Ende des zwanzigsten Jahrhunderts wurde deswegen als ein wichtiger Schritt betrachtet, um der US-amerikanischen Wirtschaft auch bei der Entwicklung innovativer Produkte Konkurrenz machen zu können.

Nach den Kurszusammenbrüchen an der NASDAQ, dem Neuen Markt oder dem Nouvelle Marché, nach der Diskussion über $\mathrm{Bi}$ lanztricksereien bei Vorzeigeunternehmen der New Economy und der Pleite ehemaliger Starunternehmen wie Webvan in den USA, Boo.com in Großbritannien oder Kabel New Media in Deutschland scheint es an der Zeit, die Funktionsweise von Risikokapital, die Mythologie der New Economy und die Logik schnell wachsender Unternehmen genauer zu analysieren.

Der Artikel versteht sich als Beitrag zu einer Soziologie kapitalmarktorientierter Unternehmen und orientiert sich an der neoinstitutionalistisch ausgerichteten Diskussion über die Überlebensstrategien von Unternehmen. John W. Meyer und Brian Rowan (1977) haben in impliziter Abgrenzung von polit- und institutionenökonomischen Ansätzen darauf aufmerksam gemacht, dass die Steigerung der Effizienz und der Rentabilität sowie das Anwachsen des operativen Gewinns nur eine Überlebensstrategie unter vielen ist. Eine weitere Überlebensstrategie für Organisationen besteht darin, sich erfolgreich in ihre institutionelle Umwelt schreiben". Trotz ausbleibender Profitabilität im operativen Geschäft können Unternehmen so ihre Existenz sichern. Die Soziologie kapitalmarktorientierter Unternehmen widmet sich dabei besonders den Rationalitätsmythen und -annahmen, die am Kapitalmarkt zur Herstellung organisatorischer Legitimität gepflegt werden.

In diesem Artikel soll gezeigt werden, wie institutionelle Legitimität während der New Economy in Geldzahlungen an Unternehmen übersetzt wurde und welche Wandlungs- und Veränderungsprozesse dem zugrunde liegen. Das konzeptionelle Hauptproblem des NeoInstitutionalismus besteht darin, dass er nur begrenzt in der Lage ist, Veränderungen von Institutionen zu erklären, was sich auf das Fehlen eines systematisch entwickelten Akteursbegriffs zurückführen läßt. Der Akteursbegriff dient anderen Theorien, wie der Institutionenökonomie, der Mikropolitik oder auch Teilen der Politökonomie, dazu, die Entstehung und Veränderung von Institutionen über die Eigeninteressen der Akteure oder das häufig konfliktbeladene $\mathrm{Zu}$ sammenspiel von Akteuren zu erklären.

Dieser Beitrag hat nicht den Anspruch, diese konzeptionelle Lücke im Neo-Institutionalismus theoretisch zu schließen (siehe dazu Walgenbach 2002). Vielmehr sollen am Beispiel der New Economy neoinstitutionalistische Interpretationen vorgeschlagen werden, die explizit oder implizit den Wandel von Institutionen betreffen. Dabei lässt sich zeigen, wie neue Annahmen bezüglich Marktbedingungen zu einer Veränderung der Legitimitätsanforderungen an Unternehmen geführt haben. Der Glaube an eine grundlegend neue Funktionsweise der Wirtschaft (Bedeutung von Transaktionskostensenkung, Lock-ins, Skaleneffekte) hat dazu geführt, dass am Kapitalmarkt schnell wachsenden Unternehmen besondere Überlebenschancen zugestanden und dementsprechend liquide Mittel zur Verfügung gestellt wurden.

Eine zentrale These des Artikel ist, dass "Legitimitätsvorteile", die im Neo-Institutionalismus in der Regel etablierten Unternehmen zugeschrieben wurden (z.B. Meyer/ Zucker 1989), auch für neue Unternehmen gelten können (Abschnitt 1). Diese ,Legitimitätsvorteile" müssen so strukturiert sein, 
dass sie sich für Unternehmen in Form von Geldzahlungen ausdrücken. Der Neo-Institutionalismus hat herausgearbeitet, dass sich Unternehmen allein von einer hohen Legitimität in ihrer institutionellen Umwelt noch nichts kaufen können. Vielmehr muss diese Legitimität in Steuernachlässe, Subventionen, Zahlungserleichterungen und Kredite umgewandelt werden, damit die Unternehmen ihre Zahlungsfähigkeit aufrechterhalten können (vgl. Meyer/Rowan 1977). Erst die Institutionalisierung der Risikokapitalfinanzierung seit dem Zweiten Weltkrieg hat bewirkt, dass Legitimitätsvorteile in der institutionellen Umwelt auch von jungen Unternehmen in Geldzuwendungen übersetzt werden können. Die Orientierung an „First Mover Advantages", Marktführerschaft und rascher internationaler Expansion fübrte dazu, dass sich Unternehmen bereits in ihrer frühen Phase als ideales Investitionsfeld für Risikokapitalgeber präsentieren konnten (Abschnitt 2). Der Verdienst des Neo-Institutionalismus besteht darin, aufgezeigt zu haben, dass Unternehmen in der Regel nicht nur mittels einer profitablen Produktion überleben können, sondern auch, indem sie in ihrer institutionellen Umwelt Legitimität erzeugen, mit der sie teilweise defizitäre Geschäfte ausgleichen können. Doch ist diese in Geldzahlungen umgewandelte institutionelle Legitimität nicht voraussetzungslos. Genauso wenig wie defizitäre Stahlkonzerne, Zeitschriften und Fleischverpackungsunternehmen von der institutionellen Umwelt bedingungslos Geldzahlungen bekommen, erhalten kapitalmarktorientierte Unternehmen Geld „einfach so“. Vielmehr werden Kriterien jenseits der Profitabilität ausgebildet, an denen die Unternehmen gemessen werden können. Bei den Internetunternehmen waren dies die Anzahl von Nutzern und Bildschirmbesuchern, die "Foot prints" im internationalen Markt sowie gesicherte Patente und Umsatzsteigerungen (Abschnitt 3). Erfolg und Misserfolg von Internetunternehmen waren maßgeblich vom Nachfluss von Investitionskapital durch Business Angels, Risikokapitalgeber oder die Aktienausgabe an Börsen abhängig. Dieser Geldfluss hing davon ab, ob Firmen Meilensteine in Bezug auf $\mathrm{Be}$ nutzerzahlen, Umsätze oder später auch Un- ternehmensergebnisse erreichten. An dieser Stelle setzt in vielen Unternehmen eine Entkopplung zwischen den nach außen zum Zweck der Legitimitätssicherung präsentierten Zahlen und der „Betriebsrealität", also den technisch ablaufenden Prozessen, ein. Unternehmen der New Economy wandten alle möglichen Tricks an, um die entsprechenden Signale an den Kapitalmarkt senden zu können. Wenn die Entkopplungen offensichtlich wurden und deswegen diese Signale nicht mehr gegeben werden konnten, brach das auf permanente Nachflüsse vom Kapitalmarkt basierende Geschäftsmodell häufig in sich zusammen (Abschnitt 4). Kapitalund Produktmarktorientierung hängen insofern zusammen, als sich Risikokapitalanleger theoretisch an der langfristigen Profitabilität von Unternehmen orientieren. Da aber die Anteile an Wachstumsunternehmen ein eigenes Handelsgut darstellen, kann es zu einer Entkopplung von Produktmarkt und Kapitalmarkt kommen (Abschnitt 5). ${ }^{1}$

\section{Die Spezifik des Internet-Hypes und die Folgen für die strategische Orientierung von Firmen der New Economy}

Im Zeitraum von 1995 bis 2000 überstieg die Kapitalmarktbewertung von Internetfirmen häufig die Marktkapitalisierung großer, etablierter Old Economy-Unternehmen. Priceline, ein Unternehmen, das Flugtickets, Mietautos und Hotelbetten im Internet anbietet, erreichte beispielsweise trotz hoher Verluste eine Marktkapitalisierung in Höhe von 21 Milliarden US-Dollar und war damit ,mehr wert" als die Fluggesellschaften Delta Air Lines, US Airways und United Airlines zusammen (Foust 1999: 58). Der InternetBuchhändler Amazon.com erreichte mit einer Marktkapitalisierung in Höhe von 24 Milliarden US-Dollar zeitweise einen höheren Wert als alle anderen börsennotierten Buchhandelsketten der USA, einschließlich Barnes \& Nobles und Borders (vgl. Himelstein 1999: 59).

Die hohe Marktkapitalisierung der Internetunternehmen lässt sich darauf zurück- 
führen, dass in einem bis dahin ungekannten Maße Risikokapital in junge Unternehmen investiert wurde. Die hohen Preise, die bei Erstemissionen von teilweise erst ein oder zwei Jahre alten, hoch defizitären Unternehmen bezahlt wurden, das starke Ansteigen der Kurse von Internetunternehmen an der Börse und die hohe Bewertung von Business-Plänen der Unternehmen durch Risikokapitalgeber sind Indizien dafür, dass es sich beim Internet um einen Geschäftszweig handelte, der wie kaum ein anderer Markt risikobereite Investoren anzog. Es stellt sich nun die Frage, welche Überlegungen hinsichtlich der Wirtschaft in der ,Informationsgesellschaft" die hohe Bewertung der Internetfirmen nährten - oder theoretischer formuliert: Aus welchen Elementen muss ein $\mathrm{My}$ thos bestehen, der es jungen Unternehmen ermöglicht, eine hohe Legitimität in der institutionellen Umwelt zu erzielen?

\subsection{Das Internet als treibende Kraft einer Effizienzsteigerung in allen}

Wirtschaftsbereichen

Das Wort „Revolution“ genügte den meisten Beobachtern kaum, um die Entwicklung der "digitalen Ökonomie" (Tapscott 1996), der "E-conomy" (Hartman/Sifonis 2000) oder der "Net-Economy“ (Hagel/Armstrong 1997) zu beschreiben. Don Tapscott, einer der ersten Verkünder revolutionärer Umwälzungen, vermeldete, dass die „ersten, turbulenten Tage einer Revolution" $z$ u beobachten seien, die möglicherweise alle anderen "Revolutionen in den Schatten stellen" werde. Ein ,neues Medium menschlicher Kommunikation" sei am Entstehen, das eine "neue Wirtschaft" ermögliche, die auf der "Vernetzung der menschlichen Intelligenz" basiere (vgl. Tapscott 1996: xv). Kevin Kelly, einer der Herausgeber des Magazins Wired und als "Guru" der New Economy gefeiert, behauptete, dass die New Economy grundlegend „neue Regeln" hervorbringe, die das Geschäft vieler Unternehmen auf den Kopf stellen werde (Kelly 1998: 1ff.).

Der Journalist Jeffrey M. Laderman bezeichnete das Internet als „das Instrument des Informationszeitalters", Genauso wie die
Eisenbahn im neunzehnten und das Auto im zwanzigsten Jahrhundert revolutioniere heute das Internet die Art und Weise, wie wir arbeiten, leben und Geschäfte machen (Laderman 1999: 52). Der Bau von Eisenbahnnetzen im neunzehnten und von Autostraßen im zwanzigsten Jahrhundert habe, so der in der Internetwirtschaft zu hörende Vergleich, den Transport von Gütern und Personen einfacher, billiger und effizienter gemacht. Europa, Amerika, Australien und einzelne Länder in Asien und Afrika seien durch Eisenbahn und Straßen zu effizienten nationalen oder transnationalen Marktplätzen geworden, in denen Einkaufs-, Produktions- und Vertriebsprozesse revolutionär erneuert werden konnten. Durch den Aufbau eines eng geknüpften Schienennetzes war es möglich geworden, Zulieferprodukte dort einzukaufen, wo sie besonders günstig produziert wurden - auch dann, wenn der Produktionsort mehrere hundert oder gar tausend Kilometer entfernt war. Die Produktion eines Unternehmens brauchte nicht mehr an einem Ort konzentriert zu werden, sondern konnte auf verschiedene Orte verteilt werden, weil die Zwischenprodukte über die Eisenbahn kostengünstig transportiert werden konnten (vgl. Madrick 1999; Mandel 1999).

Dem Internet wurde ein ähnlicher Einfluss auf die Weltwirtschaft zugetraut. Während vor dem Internetzeitalter international vorrangig mit Gütern gehandelt worden sei, könnten jetzt Finanz-, Beratungs- und Fortbildungsleistungen weltweit angeboten und nachgefragt werden. Softwareprogrammierung, Datenprozessierung, Wartung und telefonisch oder elektronisch gestützte Kundenbetreuung könnten mit Hilfe des Internet dort vorgenommen werden, wo es am kostengünstigsten ist. Auch die Produktion materieller Güter an verschiedenen Standorten werde erleichtert, weil über das Internet die Abstimmung zwischen den verschiedenen Produktionsstandorten effizient abgewickelt werden könne.

Insgesamt, so die Annahme, mache das Internet alle Anbieter, Nachfrager und Makler vergleichbarer, was letztlich in allen Bereichen die Effizienz steigern würde. Als Ergebnis erwartete man einen höheren Grad an Produktivität in der Gesamtwirtschaft. 
Doch wer würde diesen entstehenden Markt beherrschen?

\subsection{Die Bedeutung des Lock-in}

In der New Economy herrschte bei vielen Unternehmen die Vorstellung, dass das klassische Schumpeter'sche Theorem der kreativen Zerstörung überwunden werden könne. Schumpeters Gedanke war, dass ein Unternehmen, das eine Innovation durchsetzt, kurzfristig monopolistische Preise verlangen und dadurch hohe Gewinne erzielen könne. Die Dienstleistung, das Produkt oder der Vertriebsweg werde jedoch schnell von Konkurrenten nachgeahmt, was die Gewinne sinken lasse. Produkte, Produktionsverfahren und Vertriebswege, die sich in dieser Konkurrenzsituation nicht mehr halten können, scheiden aus dem Wirtschaftsprozess aus, und nur die Unternehmen, die neue Innovationen einführen, können für kurze Zeit wieder Monopolpreise verlangen.

Im Gegensatz zu dieser Schumpeter'schen Idee sahen einige Vordenker der New Economy die großen Gewinner der Epoche unter denjenigen Unternehmen, die mit einer wachsenden Zahl von Nutzern eine Dynamik entwickeln, die fast von alleine immer neue Nutzer auf die eigenen Produkte lenken und so zu deren weiteren Verbreitung beitragen würde. Nur wer es schaffe, so die dominierende Auffassung, über hohe Anfangsinvestitionen eine ,weltweite Einheitstechnologie" zu etablieren, könne damit rechnen, langfristig Kunden zu binden. Nur wem es gelinge, eine große Anzahl von Anbietern unter seinen Portalen zu versammeln, würde immer noch mehr Anbieter zu seinen Portalen ziehen. Nur die Online-Marktplätze, die einen hohen Marktanteil erobern könnten, würden sich durchsetzen, weil die Kunden nach den optimalen Märkten suchen würden und diese nur durch eine hohe Teilnehmerzahl erreicht werden könnten.

Die zentrale Bedeutung des schnellen Überschreitens eines kritischen Punktes wurde mit dem Konzept des Lock-in begründet. Unter einem Lock-in wird ein Prozess verstanden, in dem sich durch selbstverstärkende Entwicklungen eine einmal eingeführte
Technologie, Marke oder strategische Ausrichtung immer mehr ausbreitet. Es entsteht ein Standard, von dem sich Beteiligte nur noch mit hohen Kosten lösen können (vgl. grundlegend Arthur 1994). Die Faszination des Lock-in besteht darin, dass der Besitzer aus ,,seinem“ Standard enorme Profite ziehen kann (vgl. Evans/Wurster 2000: 35). Daher kann es sich langfristig lohnen, Produkte und Dienstleistungen umsonst abzugeben, um so möglichst viele Nutzer anzulocken, erfolgreich einen Standard zu etablieren und dann allmählich Geld für die Leistungen zu verlangen (vgl. Hartman/Sifonis 2000: 92ff.).

Der Geschäftsführer eines Unternehmens, das sich auf die Beratung von Internet-Startups konzentriert, erklärt, dass die Strategie der Unternehmen immer gleich gewesen sei. „Man hat eine Idee, die stellt man ins Internet: da passiert irgend etwas, ich kann etwas kaufen, ich kann etwas verkaufen, ich kann handeln oder ich kriege Börseninformationen." Das mache man am Anfang immer umsonst. Der Plan sei dabei aber immer „viele, viele User zu kriegen“, denen man dann irgendwann verkünden könne, „das kostet jetzt Geld." Die Hoffnung sei, dass man bis dahin eine so hohe User-Zahl erreicht habe, dass die Kunden es nicht mehr riskieren könnten, auf die Dienstleistung zu verzichten (Unternehmen A, Vorstandsvorsitzender und Gründer).

Die Kunden, so die Überzeugung, gingen nicht allein deswegen auf die Seiten von eBay, weil dort so attraktive Leistungen angeboten würden, sondern der vielen anderen Kunden wegen, die das Handeln, Kaufen und Informationen-Austauschen dort besonders interessant machen. Buchkäufer gingen zu Amazon, weil sie dort die größte Anzahl von Kundenbewertungen und die zuverlässigsten Bestsellerlisten fänden (vgl. Hagel/Armstrong 1997: 49).

\subsection{Die Spekulation auf Skaleneffekte}

Der Reiz dieses auf Lock-ins ausgerichteten Geschäftsmodells wurde durch Erwartungen bezüglich der Entwicklung von Herstellungskosten verstärkt. Die Hoffnung der Internetunternehmen bestand darin, den Lock-in der 
Kunden gut ausbeuten zu können, da bei wachsender Nutzerzahl die Herstellungskosten pro Kunde sehr stark sinken. Die Herstellungskosten eines Produkts oder einer Dienstleistung in der New Economy sind zwar anfangs sehr hoch, doch wenn das Produkt erst einmal in einer Kopie vorliegt, so die Annahme, tendieren die Kosten für weitere Kopien gegen Null.

Wenn es fünf Millionen Dollar kostet, ein neues Softwareprogramm zu schreiben, belaufen sich die Kosten für die erste Kopie auf fünf Millionen Dollar. Die Herstellungskosten für jede weitere Kopie seien dann aber quasi Null, weil sich die Kunden - gegen ein Entgeld - das Programm selbst aus dem Internet herunterladen können. Wenn 20 Millionen Euro an Werbekosten nötig wären, um die ersten Kunden zu einem Internetportal wie Yahoo, Netscape oder AOL zu locken, so gingen doch die Kosten für die Gewinnung weiterer Kunden gegen Null, weil diese allein durch Mund-zu-MundPropaganda auf die Seite fänden und irgendwann aufgrund des Lock-in eines Programms auch gar nicht mehr darum herumkämen, dieses zu nutzen (vgl. Mandel 2000: 77f.),

Dies, so die Argumentation, stelle die Funktionsweise der Wirtschaft auf den Kopf: Während in der Old Economy die massenweise Produktion von Produkten nur begrenzte Kostenersparnis bringe, weil Einkaufspreise, Herstellungspreise und Vertriebskosten sich dadurch nur um wenige Prozent reduzierten, nähmen im Internetzeitalter die Produktionskosten pro Einheit mit zunehmender Größe des Marktanteils radikal ab. Ein Softwareprogramm könne innerhalb von Stunden und Tagen an Millionen von Kunden verteilt werden, ohne dass, wie etwa in der Automobilindustrie, für jedes neue Exemplar erneut in Material, Löhne und Vertrieb investiert werden müsse.

Zur Boom-Zeit der New Economy war in den Business-Plänen der Internetunternehmen, in den PowerPoint-Präsentationen der Berater und in den Artikeln der Wirtschaftszeitschriften von der "revolutionären" Reduzierung der Kosten für Finanztransaktionen die Rede. Während eine Finanztransaktion in einer Bankfiliale einen Dollar, am
Cent koste, entstünden beim InternetBanking nur noch Kosten in Höhe von einem Cent. Die Entwicklung eines Internetportals stelle aus dieser Perspektive zwar eine erhebliche Investition dar, würde sich aber bei wachsender Kundenzahl auszahlen. Die Internetbanken hätten einen so großen Kostenvorteil gegenüber klassischen Banken, dass sie diesen durch den Preiswettbewerb gefährlich werden könnten (vgl. Farrell 1999: 72; The Economist, 11.11.2000: 115).

\subsection{Die Rolle von Mythen}

Die oben skizzierten Annahmen über die Beeinflussung der Weltwirtschaft durch das Internet, über die Bedeutung von Lock-ins und über explosionsartige Skaleneffekte können insofern als Mythen der Frühzeit der New Economy gelten, als sie in ihrer Funktion als Handlungsgrundlage kaum noch hinterfragt wurden. Sie wurden zu Rationalitätsannahmen, an denen innerhalb der NewEconomy-Szene kaum gerüttelt werden konnte, und dominierten Business-Pläne, Geschäftsberichte wie auch die obligatorischen Prospekte zu Börsengängen.

Ein Großteil der Investoren, der Medien und der Unternehmen nahm diese Vorstellungen als Entscheidungs- und Handlungsprämissen hin, ohne dass es zureichende Beweise dafür gegeben hätte. Skeptische Äußerungen etwa darüber, dass das Internet lediglich eine neue Kommunikationsform neben Telefon, Fax und Brief darstelle, Produktivitätssteigerungen durch Computer, Software oder Internet noch nicht bewiesen seien, dass das Internet Lock-ins eher unwahrscheinlicher mache, als dass es sie ermögliche, oder dass die Skaleneffekte so groß nicht sein würden, wurden kaum wahrgenommen. Mit Schlagwörtern wie „Now or Never" (Modahl 2000), "Get Net Ready“ (Hartman/Sifonis 2000) oder „The New Logic of Digital Business" (Cronin 2000) wurde suggeriert, dass Unternehmen nur die Wahl hätten, sich auf die New Economy einzulassen oder yon der Bildfläche zu yerschwinden.

Dabei darf nicht übersehen werden, dass gerade in einem höchst unsicheren Umfeld 
wie der New Economy Mythen eine wichtige Funktion erfüllen. Sie helfen, die Unsicherheit von Entscheidern zu absorbieren. Die fast religiös anmutende Verherrlichung der New Economy in Zeitschriften wie Wired, Industry Standard oder Business Week und in Büchern von Unternehmensberatern, Analysten und Unternehmern lieferte die "notwendige Ignoranz" in einem höchst unsicheren Umfeld und machte so die Akteure der New Economy entscheidungs- und handlungsfähig (Brunsson 1985). Durch die Verständigung, dass das Internet etwas "ganz, ganz Großes" und die „Entwicklung revolutionär" sei, war es Unternehmen möglich, eine Dynamik zu entwickeln, die durch zu große Fragezeichen und zu große Zweifel nur behindert worden wäre.

Bestimmte Annahmen der New Economy im Nachhinein als falsch zu entlarven, ist aus soziologischer Perspektive nur begrenzt ergiebig und verkennt die Notwendigkeit von Mythen für Organisationen, um unter Bedingungen zunehmender Unsicherheit überhaupt handlungsfähig zu bleiben. Vielmehr ist die New Economy ein Beispiel dafür, wie Annahmen über die Entwicklung der Wirtschaft dazu führen können, dass neue Organisationen einen Legitimitätsvorteil erhalten. Während der Neo-Institutionalismus sich bisher vorrangig darauf konzentriert hat, die institutionelle Legitimität von etablierten Organisationen herauszuarbeiten, wird hier deutlich, dass bei bestimmten Erwartungen hinsichtlich sich radikal ändernder Wirtschaftsbedingungen neu entstehende Organisationen einen Legitimitätsvorteil haben können. Diese Legitimitätsvorteile entstehen nicht aus deren eigener Geschichte sondern aus Wachstumspotenzialen.

\section{Das Business-Modell der Internetfirmen und die Logik des kompromisslosen Wachstums}

Die institutionelle Legitimität an sich kann noch nicht das Überleben eines Unternehmens sichern. Nur durch die rhetorische Unterstützung der Politik können hochdefizitäre Stahlunternehmen wie Krupp nicht überleben. Allein der Hinweis der Besitzer defizitärer Zeitungen wie der Welt, dass sie ihr Blatt aufgrund der politischen Ansprüche für sinnvoll halten, reicht nicht aus, um dessen Überleben zu sichern. Die Mitarbeiter der defizitären Fleischverpackungsfirma Rath Packing Company können noch so sehr die Notwendigkeit des Überlebens der Firma betonen und auf die hohe Akzeptanz in der institutionellen Umwelt verweisen, diese Legitimitätsvorteile müssen von einer rein rhetorischen Unterstützung in Geldzuflüsse übersetzt werden (Meyer/ Zucker 1989). Die Legitimitätsvorteile müssen in Form von Steuernachlässen, Subventionen, Zahlungserleichterungen und Krediten durch den Staat, durch politisch interessierte Kapitalbesitzer oder durch Kooperationspartner in Geldzahlungen an das Unternehmen konkretisiert werden.

Im Fall der hier untersuchten kapitalmarktorientierten Unternehmen findet die Übersetzung durch Risikokapitalgeber statt, die sich seit dem Zweiten Weltkrieg institutionalisiert haben. Die Konzeption der Risikokapitalgesellschaften ist in den fünfziger und sechziger Jahren in den USA entstanden und hat sich dann nach Europa, Asien und in den Nahen Osten verbreitet. Risikokapital wird vorrangig über drei Institutionen an Wachstumsunternehmen weitergegeben. In der Frühphase einer Gründung beteiligen sich häufig vermögende Einzelpersonen, so genannte Business Angels, direkt mit ihrem eigenen Kapital an einem Unternehmen. In der Wachstumsphase erwerben Risikokapitalgesellschaften, die ihr Kapital wiederum von reichen Privatpersonen, Rentenversicherungen, Banken, Großunternehmen und anderen Investorengemeinschaften erhalten, Anteile am Unternehmen. In späteren Phasen bieten Börsen für Wachstumsunternehmen, die parallel zur Ausbreitung der Idee der Business Angels und zum Anwachsen von Risikokapitalgesellschaften entstanden sind, risikokapitalfinanzierten Unternehmen eine weitere Möglichkeit, sich mit neuem Kapital einzudecken (ausführlich Kühl 2003). 


\subsection{Von First Movern und Market Leadern}

Die Erwartungen hinsichtlich hoch profitabler Lock-ins, die Hoffnung auf enorme Skaleneffekte in der Informations- und Kommunikationstechnologie und die Überzeugung von der Penetrierung der gesamten Weltwirtschaft durch das Internet konzentrierten sich im Diskurs der New Economy in einem Begriff: dem des ,First Mover". Wem es gelingen würde, als Erster ein bestimmtes Produkt oder eine bestimmte Dienstleistung anzubieten, dem würde die verstärkte Aufmerksamkeit von Kunden zuteil, er würde von Investoren belagert, umfassende $\mathrm{Be}$ richterstattung in den Medien erhalten und das Interesse der großen Unternehmen der Informations- und Kommunikationstechnologie auf sich ziehen.

Der Geschäftsführer einer Risikokapitalfirma berichtet, es habe der "Glaube" bestanden, dass der "First Mover" erfolgreich „die Kunden an sich binden kann". Er wisse nicht mehr, ,in wie vielen Business-Plänen das stand". Diejenigen, die nach einem ,First Mover" auf den Markt gekommen seien, seien "meist nicht mehr relevant" gewesen, "auch wenn das Produkt besser war" (Venture Capital Firma A, Geschäftsführer). Der Investmentmanager einer anderen Risikokapitalgesellschaft beschreibt den damals dominierenden Blickwinkel als klassische First-Mover-Logik: „Wachse, so schnell du kannst, sonst tun es andere" (Venture Capital Gesellschaft B, Investmentmanager).

Man war der Ansicht, dass ein ,winner takes all"-Geschäft oder wenigstens ein ,winner takes most"-Geschäft herrsche. Alle Unternehmen befänden sich in einem Henne-EiDilemma: Man brauche auf den Plattformen Käufer, um Verkäufer anzuziehen, und Verkäufer, um Käufer anzulocken. Das Unternehmen, dem es gelinge, diesen Teufelskreis zu durchbrechen und möglichst schnell eine kritische Masse von Verkäufern und Käufern anzuziehen, erhalte ein „Momentum", durch das fast automatisch neue Kunden dazukämen. Konkurrenten mit besseren Produkten und Preisen hätten dann kaum noch Chancen, zum Marktführer aufzuschließen (vgl. Hof 2000: 72),
Das in der New-Economy-Szene kursierende Bild war das zweier Restaurants, von denen dasjenige einen Vorteil hat, dem sich Kunden zuerst zuwenden. Wenn ein Gast die Wahl zwischen zwei nebeneinander liegenden Restaurants hat, schaut er von außen durch die Scheiben und prüft, in welchem Restaurant mehr Personen sitzen. Er entscheidet sich für das Restaurant, in dem schon jemand sitzt. Damit erhöht er den Anreiz für den nächsten Kunden, sich auch diesem Restaurant zuzuwenden. So entsteht eine Kettenreaktion, durch die sich das eine Restaurant aufgrund des kleinen Startvorteils kontinuierlich füllt, während das andere Restaurant, das den ersten Kunden nicht anziehen konnte, nur mit erheblichem Aufwand den Startnachteil wieder aufholen kann (Shiller 2000: 152). Diese aus Überlegungen zu Lock-ins und Skaleneffekten abgeleiteten Überzeugungen hatten die Konsequenz, dass Internetfirmen mit extrem hohen Wachstumserwartungen konfrontiert wurden.

\subsection{Die Wachstumslogik und das Interesse der Risikokapitalgeber}

Die Business-Modelle der Internetfirmen waren in der Boom-Zeit der New Economy von 1995 bis 2000 überall sehr ähnlich: rasantes Wachstum, schnelle internationale Expansion und möglichst nach zwei oder drei Jahren Marktführerschaft. Das Mantra der New Economy, so der Journalist Robert D. Hof, war "GBF": „Get Big Fast". Der Gründer und stellvertretende Vorstandsvorsitzende von Priceline, Jay S. Walker, beispielsweise erklärte, dass man entweder das Unternehmen "schnell groß mache“, oder „man mache es überhaupt nicht" (vgl. Hof 2001: 50).

Ein Investmentmanager, der früher als Personalmanager ein Internetunternehmen mit aufgebaut hat, erklärt, dass die ,klassische Wachstumskurve eines Internetunternehmens" darauf basiert habe, „möglichst kurzfristig, schnell und regional" $\mathrm{zu}$ expandieren. Es habe Firmen gegeben, die in „sechs bis neun Monaten ihre Größe um den Faktor zehn erhöht" haben. Ein Internetunternehmen, das ,innerhalb von sechs Mo- 
naten nicht mindestens eine europäische Niederlassung gehabt" habe, sei in der „Wahrnehmung eines Investors" schon „tot gewesen" (Venture Capital Firma B, Investmentmanager).

Die Vorbilder der New Economy waren Unternehmen wie eBay, At Home und Amazon, die ihre Umsätze pro Jahr mehr als verdreifachten. Während eine „Old Economy"-Firma wie IBM von Anfang 1997 bis Anfang 1999 ein jährliches Umsatzwachstum von acht Prozent aufzuweisen hatte, steigerte Amazon seinen Umsatz jährlich um 328 Prozent, At Home um 460 Prozent und eBay gar um über 1000 Prozent (vgl. Himelstein 1999: 59).

Die Fokussierung auf schnelles Wachstum machte die Internetunternehmen überhaupt erst für Risikokapitalgeber interessant. Der Geschäftsführer einer Risikokapitalgesellschaft stellt fest, dass kein Risikokapitalgeber in ein Unternehmen investieren würde, das ein Wachstum von nur zehn Prozent pro Jahr anstreben würde. Solch ein Unternehmen „,braucht keinen Venture Capitalist", da in seinem Fall „Bankkredite fast billiger" seien (Venture Capital Gesellschaft C, Gründer und Geschäftsführer). Wenn ein Unternehmen nicht richtig wachsen könne, so ein anderer Risikokapitalgeber, dann müsse man ihm klar sagen, dass es nicht „VC-fähig" sei (Venture Capital Gesellschaft E, Gründer und Geschäftsführer).

Das Geschäftsmodell von Risikokapitalgebern basiert darauf, dass nur einige wenige Unternehmen im Portfolio den Investoren "richtig Geld" bringen. In fast monotoner Weise wird von Risikokapitalgebern betont, dass von zehn Unternehmen nur ein oder zwei für richtig viel Geld an die Börse gebracht oder sehr teuer an andere Unternehmen verkauft werden könnten. Bei drei, vier oder fünf Unternehmen könne man hoffen, dass man die Anteile daran mit einer einigermaßen anständigen Rendite verkaufen könne oder wenigstens seine Ausgaben wieder hereinbekomme. Den Rest der Unternehmen könne man abschreiben, weil sie vor sich hin vegetierten oder Konkurs gingen. Ein Risikokapitalgeber erklärt, dass zwar von zehn Unternehmen ein, zwei oder drei ,auf alle Fälle kaputtgehen“, in der Regel aber ,ein oder zwei die richtigen Reißer" würden. Der Rest seien ,lebende Tote", die nicht schlecht liefen, aber nur schwer zu verkaufen seien (Venture Capital Gesellschaft E, Gründer und Geschäftsführer).

Dieses Kalkül bedeutet aber, dass Risikokapitalgeber nur in solche Unternehmen investieren, von denen sie hoffen können, sie für ein Vielfaches der Einstiegssumme verkaufen zu können. Lutz Krafft, Organisator des Forschungsnetzwerkes e-startup.org, erklärt, dass ein Risikokapitalgeber die Investition in ein Unternehmen ,nicht verdoppeln, nicht verdreifachen, sondern wenn möglich verzehnfachen" wolle. Das bedeute, dass ein Unternehmen eine Anschubfinanzierung von einer Million nur dann bekomme, wenn der Risikokapitalgeber eine Perspektive sehe, nach einigen Jahren fünf bis zehn Millionen wieder zurückzubekommen. Wenn ein Unternehmen dieses Wachstumspotenzial nicht hergebe, brauche man sich gar nicht erst an einen Risikokapitalgeber zu wenden (vgl. Krafft 2002: 2).

\subsection{Die Institutionalisierung der Risikokapitalszene und die Mythen der New Economy}

Die Argumentation, dass Internetunternehmen ein ideales Investitionsfeld für Risikokapitalgeber waren, lässt sich drehen: Erst durch die Institutionalisierung der Risikokapitalfinanzierung seit dem Zweiten Weltkrieg und der wegen der Risikostreuung notwendigen Fokussierung der Risikokapitalgesellschaften auf schnell wachsende Unternehmen konnten die Erwartung hinsichtlich Lock-ins, Skaleneffekten und Transaktionskostensenkungen zu der Gründungswelle der New Economy führen. In der New Economy hatten wir es mit der seltenen Kombination aus dominanten Mythen über die zukünftige Funktionsweise der Wirtschaft und in Überschuss zur Verfügung stehenden Finanzmitteln für schnell wachsende Unternehmen zu tun, die zu der Möglichkeit führte, dass sich junge Unternehmen aufgrund ihrer hohen Legitimität in der institutionellen Umwelt mit Kapital versorgen konnten. 
Dabei lässt sich - wenigstens als Hypothese - von einem rekursiven Verhältnis zwischen der Mythenproduktion und der Risikokapitalfinanzierung ausgehen. Mythen sind ,nicht einfach so in der Welt", sondern sie müssen plausibilisiert werden. Risikokapitalgeber beziehen sich auf die Rationalitätsannahmen über die Veränderung der Wirtschaft, tragen aber mit der über sie zur Verfügung gestellten Finanzierung zur Plausibilisierung dieser Mythen bei. Über Risikokapitalgesellschaften stehen Gelder zur Verfügung, mit denen Unternehmen auf die Mythen bezogene „Erfolgsgeschichten“ schreiben können, die aber für mehrere Jahre nicht durch Profitabilität im Kerngeschäft „verifiziert" werden müssen.

\section{Nutzerzahlen, Expansion und} Steigerung des Umsatzes: Die Erfolgskriterien für Firmen der New Economy

In der Hochzeit der New Economy war Profitabilität kein überzeugendes Argument für Investoren. Im Gegenteil: Der Geschäftsführer einer Venture Capital Firma erklärt, dass es ,richtig negativ" gewesen sei, wenn ein Unternehmen Gewinne aufweisen konnte. „Aus damaliger Sicht war klar, dass das Internet einen ziemlich großen Anteil an dem Weltwirtschaftsprodukt" haben würde. Die Frage: „Mache ich jetzt dieses Quartal kurzfristig Gewinn oder nicht?", habe keine Rolle gespielt. Im Mittelpunkt habe die Frage gestanden: „Wie groß wird der Marktanteil meines Unternehmens auf diesem explodierenden Markt?" Wenn ein Unternehmen zu wenig Geld ausgegeben habe, dann sei ihm gesagt worden: „Ihr gebt nicht genügend Geld aus, um den Marktanteil zu erobern, und langfristig seid ihr damit tot." „Profit", so der Risikokapitalgeber, „ist überhaupt nicht ausschlaggebend gewesen". Interessiert habe nur, "welchen Marktanteil ich habe", "wie viele Click-Rates" ich vorweisen kann (Venture Capital Firma A, Gründer und Geschäftsführer).

Aufgrund dieser Einstellung wurden die
Werts eines Unternehmens wie das Kurs/ Gewinn-Verhältnis oder die Dividende wertlos. Das Kurs/Gewinn-Verhältnis ist bei Unternehmen, die lediglich Verluste ausweisen, nicht zu bestimmen. Und wenn das Unternehmen keinen operativen Gewinn macht, gibt es auch nichts, woraus Dividenden bezahlt werden könnten.

In der New Economy bildeten sich daher Bewertungsmaßstäbe aus, die jenseits der Vorstellungen von Kurs/Gewinn-Verhältnissen und Dividenden lagen. Die Bewertungsmaßstäbe waren dabei zuerst auf „User" und das "geistige Kapital" konzentriert, dann aber zunehmend auf das Kriterium der Umsatzsteigerung. Dieser Prozess ist neoinstitutionalistisch interessant, weil bisher von dieser Theorierichtung nur begrenzt herausgearbeitet wurde, wie die eher diffusen institutionellen Legitimitätserwartungen in konkrete Erwartungshaltungen in Organisationen übersetzt werden und in welcher Form sich diese verändern können.

\subsection{Bildschirmbesuche und Benutzerzahlen}

Eyeballs, Page Impressions, Click-Rates, Registered Users und Mindshare wurden in der New Economy als wesentliche Leistungskriterien betrachtet. Unter der Bezeichnung Eyeballs werden die Personen gezählt, die sich eine Website ansehen. 25.000 Eyeballs im Monat bedeutet, dass 25.000 Personen diese Website aufgerufen haben. Unter Page Impressions wird festgehalten, wie lange ein Internetsurfer bei einem Anbieter bleibt bzw. durch wie viele Einzelseiten einer Website er sich klickt. Wenn sich ein Nutzer sieben Einzelseiten eines Anbieters ansieht, hinterlässt er auf dem Surfer eine Page Impression von sieben. Click-Rates geben das Verhältnis zwischen Sichtkontakten und tatsächlich angeklickten Informations- oder Werbebannern wieder. Damit kann gemessen werden, welche der Dutzende von Links und Schaltflächen auf einer Internetseite besonders häufig genutzt werden. Eine Click-Rate von fünf bedeutet, dass von 100 Leuten, die einen Link, eine Schaltfläche oder ein Banner gesehen haben, 
fünf darauf geklickt haben. Mit der Kategorie Registered Users wird gezählt, wie viele Personen sich auf einer Seite angemeldet und dabei Informationen über sich hinterlassen haben. Unter dem Begriff Mindshare schließlich wird angegeben, wie bekannt eine Marke unter Internetusern ist.

Die starke Betonung von Eyeballs, Page Impressions, Click-Rates, Registered Users und Mindshare hăngt damit zusammen, dass in der Anfangszeit der New Economy davon ausgegangen wurde, dass sich die meisten Internetfirmen über Werbung finanzieren könnten. Da für die Finanzierung über Werbung, ähnlich wie bei Zeitschriften und Zeitungen, die Anzahl der Nutzer entscheidend ist, richtete sich das Interesse von Internetfirmen, Medien und Investoren anfangs auf die Anzahl der Seitenbesucher und der eingeschriebenen Benutzer. Die Erfolgsmeldungen vieler Internetfirmen bestanden darin, wie viele neue Benutzer sie jeden Tag hinzugewonnen hatten und wie stark sich ihre Click-Rate erhöht hatte.

Ann Winblad, eine der Geschäftsführerinnen der Risikokapitalgesellschaft Hummer Winblad Venture Partners, erklärt, dass sich im Internetzeitalter die „Bewertungsmaßstäbe des Erfolgs“ verändert hätten. ,Seitenbesuche insgesamt, die Anzahl einzelner Besucher und die Anzahl von Kunden wurden zum Bewertungsmaßstab" bei der "Landgewinnung im Internet". "Sogar die Banker" hätten „die Profitabilität vergessen“, und nur noch gerufen: „Gewinnt das Land!“, und sie hätten die Firmen gedrängt, Kapital dafür zu nutzen, Click-Rates und Benutzerzahlen zu steigern (Winblad 2001: 7). Mary G. Meeker entwickelte Bewertungsmaßstäbe, durch die entschieden werden konnte, welcher Kapitalmarktwert dem Nutzer einer Software oder dem Kunden eines Internet-Service-Providers wie AOL, Freeserve oder T-Online zuzuschreiben ist. Die Einschätzung des Marktwerts eines Kunden mit bis zu 10.000 Dollar basierte auf der Überlegung, dass der Kunde dem Unternehmen zwar jetzt noch kein Geld brachte, die zukünftigen Einkünfte durch den Kunden aber jene Summe übertreffen könnten (Green 1999: 35).

Diese auf Kunden oder Nutzer ausgerichtete Berechnungsform erlaubte es, den Bör- senemissionspreis von World Online International, deren Kunden mit durchschnittlich 6.500 Dollar bewertet wurden, wesentlich höher festzulegen als den des französischen Konkurrenten Liberty Serve, dessen Kunden lediglich mit 3.000 Dollar eingeschätzt wurden (vgl. Echikson 2000: 22). Mit „Digitalytics“ wurden Kapitalbewertungsmaßstäbe entwickelt, mit denen man berechnen konnte, mit wie viel Dollar der Besucher einer Website jeweils bewertet war. So konnte man beispielsweise feststellen, dass der Internet-Suchmaschine Yahoo bei 31 Millionen monatlichen Besuchern und einer Marktkapitalisierung von knapp 30 Milliarden Dollar jeder Nutzer knapp 950 Dollar Marktkapitalisierung brachte, während beim Konkurrenten Lycos mit 29 Millionen Nutzern und einer Marktkapitalisierung von nur knapp vier Milliarden Dollar jeder Nutzer als nur 135 Dollar wert betrachtet wurde. Mit Hilfe dieser Zahlen konnte man überlegen, ob der First-Mover-Vorteil von Yahoo wirklich eine sechsfache Bewertung jedes Users rechtfertigte, und sein Anlageverhalten danach ausrichten (vgl. Harmon 2001: 165).

\subsection{Patente, Programme und Potenziale:} Die Betonung des geistigen Kapitals

Ein weiteres Kriterium zur Einschätzung des Werts von Internetunternehmen war, wie sich das ,geistige Potenzial“, die ,immateriellen Werte" des Unternehmens entwickelten. Denn für ein Unternehmen ist es, so die Auffassung, nicht nur wichtig, wie viele Maschinen, Gebäude oder Cash es besitzt, sondern auch, über welche immateriellen Werte es verfügt, die sich in Forschungs- und Entwicklungsarbeiten, Softwareprogrammen, Patenten und Markennamen ausdrücken. Der Bereich der immateriellen Werte wurde als das ,zukünftige Schlachtfeld“ der Innovationen ausgemacht. Unternehmen ließen sich ihre Forschungen, Geschäftsmodelle, Marken und Softwareprogramme durch Copyrights, Patente und Markenrechte vor "Copycats", simplen Nachahmern, schützen (vgl. z.B. Davis/Meyer 1998; Tapscott/ Ticoll/Lowy 2000). 
Der Wert von Unternehmen wurde in der Hochzeit der New Economy daran gemessen, welche Patente, Marken oder Softwaremonopole ein Unternehmen hatte. Amazon ließ sich ein Patent für die Methode ausstellen, mit der man einen Buch- oder CD-Kauf mit ,einem Klick" vornehmen kann. E-Data, ein Softwareunternehmen, lie $\beta$ sich den Verkauf von Softwareprogrammen und anderen elektronischen Gütern durch das Herunterladen aus dem Internet patentieren und verklagte Dutzende von Unternehmen, die Kunden ebenfalls anboten, Programme gegen Bezahlung aus dem Internet herunterzuladen. Dem Unternehmen Priceline gestand das U.S. Patent and Trademark Office zwei Patente für sein Geschäftsmodell einer "käufergetriebenen Handelsplattform" zu. Dabei handelt es sich um eine Internetplattform, auf der Kunden einen Preis nennen können, für den sie ein Flugticket, ein Hotelzimmer, ein Mietauto oder Hypothekenkredite übernehmen würden. Unternehmen können dann erwägen, ob sie dem Käufer das nachgefragte Produkt zu diesem Preis zur Verfügung stellen wollen.

Auch wenn es unter Experten umstritten war, ob man die Internetanwendung eines bekannten Geschäftsmodells überhaupt patentieren lassen kann, interessierten sich eben aufgrund solcher Patente viele Anleger für die Aktien von Unternehmen wie Amazon, Priceline oder E-Data. Amazon warb auch bei Investoren damit, dass es durch das patentgeschützte „One Click"-Einkaufsmodell einen Vorsprung gegenüber der Konkurrenz besäße. Der Gründer von Priceline, Jay Walker, wurde nicht müde, die Marktkapitalisierung seines Unternehmens von zeitweise 24 Milliarden US-Dollar mit den zwei Patenten für sein Marktmodell zu rechtfertigen (vgl. France 1999: 90; Brady 1999: 22).

Michael Malone entwickelte eine Bilanzierungsform, mit der das geistige Kapital von Unternehmen erfasst werden sollte, bedauerte aber, dass in solche Bilanzen zwar Patente oder der Bildungsstand der Mitarbeiter eingehen, andere "weiche Faktoren" wie die Moral der Angestellten, die Dynamik der Firmenleitung oder eine innovative Umgebung jedoch noch nicht erfasst werden. wie Anlagen, Gebäude oder Fahrzeuge wird bei dieser Methode nicht mehr wie in der klassischen Bilanzierungsform auf der Habenseite notiert, sondern vielmehr als Betriebskosten auf der Sollseite geführt (Malone 1997: 40; vgl. Rifkin 2000: 74).

\subsection{Die Bedeutung des Umsatzes}

Die Fokussierung auf Click-Rates, Benutzerund Besucherzahlen basierte auf der Annahme, dass Geld im Internet durch das direkte Geschäft mit den Endverbrauchern verdient wird. In diesem Punkt stellten sich aber zunehmend Zweifel ein: Es wurde deutlich, dass es eines extrem hohen Marketing-Etats bedurfte, um Endverbraucher an eine Website zu binden. Auch gab es kaum geeignete Zahlungsmittel, mit denen Endkunden für kleinere Leistungen im Internet bezahlen konnten. Zudem verbreitete sich zunehmend die Überzeugung, dass der Werbemarkt nicht genügte, um ein Unternehmen komplett zu finanzieren.

In dieser Situation verlagerte sich das Geschäftsmodell vieler Internetfirmen - und damit auch die Bewertungsmaßstäbe - weg von den Endverbrauchern hin zu Geschäften mit anderen Unternehmen. Der Geschäftsführer einer Internetfirma berichtet, „Business-to-Business" sei die "große neue Geschichte" gewesen und als der ,neue Weg für alle" ausgerufen worden. Das Problem seines Unternehmens sei gewesen, dass sie zu wenig „im Markt drin gewesen" seien, um das Produkt im Endkundenbereich anzubieten, und dass man nicht mehr die Möglichkeit gehabt habe, bei jeder Gelegenheit ,zwanzigtausend" in die Hand zu nehmen, um ,überhaupt irgendwo gehört zu werden". So sei man, wie die meisten Internetfirmen, zu einem bestimmten Zeitpunkt nicht mehr in der Lage gewesen, sich mit einer „Kundenzahl im Werbemarkt zu positionieren", mit der man die Kosten hätte decken können. Man habe dann keine andere Möglichkeit gehabt, als von einer auf Endkunden ausgerichteten "Service-Gesellschaft" zu einer "SoftwareGesellschaft" zu werden, die Softwareprodukte an andere Unternehmen verkaufte, die dann Endverbraucher über diese Soft- 
ware auf ihre Seiten locken konnten (Unternehmen E, Gründer und Vorstandsmitglied).

Der Abteilungsleiter Technik eines Internetunternehmens erzählte, dass nach Eintreten der Erkenntnis, dass der Werbemarkt für die meisten Unternehmen nicht genug Finanzierungspotenzial hatte, viele Geschäftsmodelle, die ,ganz klar auf Business-to-Consumer (B2C) ausgelegt waren, auf Business-to-Business (B2B) umgebogen" wurden. Auch sein eigenes, eindeutig auf den Endverbraucher ausgerichtetes Unternehmen habe sich plötzlich gezwungen gesehen, ins Business-to-Business-Geschäft umzuschwenken, und habe dann eine eigene Abteilung aufgebaut, die versuchte, Serviceleistungen an andere Unternehmen zu verkaufen. Man sei „wie Lemminge" einem neuen Geschäftsmodell hinterhergerannt (Unternehmen A, Abteilungsleiter Technik).

Mit der zunehmenden Hinwendung zu Business-to-Business verloren die Seitenbesucher- und Nutzerzahlen an Bedeutung, und eine andere Zahl geriet verstärkt in den Fokus des Interesses, die auch zuvor bereits eine nicht ganz unbedeutende Rolle gespielt hatte: der Umsatz.

\subsection{Der Drang zur Quantifizierung}

Durch die Annahme, dass kurzfristige Profitabilität kein geeignetes Kriterium zur Beurteilung von Wachstumsunternehmen ist, entstand im organisationalen Feld der New Economy Unternehmen eine Unsicherheit, nach welchen Kriterien Wachstumsunternehmen überhaupt beurteilt werden können. Angesichts der Erosion existierender Profitabilitätskriterien bestand eine große Bereitschaft, neu ins Spiel gebrachte Kriterien zu übernehmen oder wenigstens zu prüfen. Aufgrund der hohen Beurteilungsunsicherheit schien das Motto zu herrschen „Lieber neuen Kriterien eine Chance geben als ganz ohne Kriterien dastehen".

Die Kriterien mussten mit Annahmen über die zukünftige Wirtschaftsentwicklung (Lock-in, First-Mover, schnelles Wachstum etc.) kompatibel und zugleich möglichst quantifizierbar sein. Schon Peter Walgenbach (2000) hat in seiner Arbeit über die „,normgerechte Institution“ festgestellt, dass die Quantifizierbarkeit auch so genannter weicher Faktoren eine zunehmend wichtige Rolle in Unternehmen spielt. Durch die Quantifizierbarkeit und damit die Messbarkeit erhalten die Angaben einen Anschein von Objektivität, der ihnen eine zusätzliche Bedeutung verleiht.

\section{Das „Herausputzen“ des Unternehmens für Investoren}

Die hohe Bewertung der Unternehmen an der Börse oder, im früheren Stadium, bei den Verhandlungen mit Risikokapitalgebern führte dazu, dass die Unternehmen sich gezwungen sahen, sehr schnell zu wachsen. Nur durch dieses Wachstum konnten sie das Image eines zukünftigen Weltmarktführers oder wenigstens regional erfolgreich aufgestellten Unternehmens nach außen verkaufen und so die hohe Bewertung rechtfertigen.

Dhiren Shah von der Investmentbank Morgan Stanley Dean Witter verkündete auf dem Höhepunkt des Internet-Hypes im März 2000, dass „Unternehmen mit einer extrem hohen Bewertung extrem hohe Wachstumsraten aufweisen müssen, um ihre Investoren zu befriedigen" (vgl. Capell 2000: 22). Der Investmentmanager eines deutschen Risikokapitalgebers stellte fest, dass die Unternehmen, die an den neuen Markt gegangen seien, eine „horrende Planung“" ihres Wachstums vorgelegt hätten. Selbst größere Unternehmen hätten ein „Wachstum von bis zu 200 Prozent pro Jahr" angestrebt. Nur durch diese Zahlen hätten sie die extrem hohe Bewertung rechtfertigen können, seien dadurch aber auch in den „Zugzwang geraten“, diese Zahlen zu erfüllen (Venture Capital Gesellschaft $\mathrm{D}$, Investmentmanager).

Die Unternehmen der New Economy waren durch die hohen Kapitalbewertungen in einer "Wachstumsfalle“ gefangen. Sie waren gezwungen, international weiter zu expandieren und ihr Produktspektrum auszudehnen, weil sie sonst ihre hohe Marktkapitalisierung nicht hätten rechtfertigen können. Aber nur die hohe Marktkapitalisierung gewährleistete, dass sie als Unternehmen mit 
keinem oder nur geringem Gewinn weiter Kapital einholen konnten. Da ihr Überleben im Gegensatz zu primär produktmarktorientierten Unternehmen davon abhing, dass sie regelmäßige Nachfinanzierung über den Kapitalmarkt bekamen, war die Aufrechterhaltung der Wachstumslogik von zentraler Bedeutung.

Die Wachstumsfalle für risikokapitalfinanzierte Unternehmen führte dazu, dass diese ihrer institutionellen Umwelt (Kapitalmarkt, Medien, Politik) permanent Meldungen über eine Steigerung von Nutzerzahlen, Mitarbeiterwachstum, Umsatzsteigerungen und Ergebnisverbesserung liefern mussten, um die institutionelle Legitimität aufrechtzuerhalten. Die Unternehmensstrategien der Firmen der New Economy enthielten einen Anreiz, die Kriterien, die ihre „Performance" bewiesen, zu erfüllen. Es bestand eine Verlockung, ,Schaufensterdekorationen“"zu kreieren, um die nächsten Finanzierungsschritte zu nehmen. Dieser Prozess wird im NeoInstitutionalismus als „Entkopplung" bzw. "lose Kopplung" bezeichnet. Meyer und Rowan (1977) betonen (im Unterschied zum Beispiel zu DiMaggio/Powell 1991), dass sich die Unternehmen zwar in ihrer Formalstruktur angleichen, ihre Kernprozesse aber von diesen Formalstrukturen entkoppelt werden können. Über den Begriff der „Entkopplung" oder ,losen Kopplung" greift der Neo-Institutionalismus die alte Unterscheidung von Formalität und Informalität auf und liefert eine Begründung, warum eine Trennung von organisatorischer Vorder- und Hinterbühne für das Unternehmen funktional sein kann (vgl. die Theoriediskussion bei Huchler 2002: 22ff.).

\subsection{Das Manipulieren von „Eyeballs“,} „Page Impressions" und „Click-Rates“

Da die Leistung eines Internetunternehmens anfangs an „Eyeballs", „Page Impressions", "Click-Rates" und „Registered Users" gemessen wurde, Kapitalgeber ihre Investitionen teilweise von der Performance in diesen Kategorien abhängig machten und bei $\mathrm{Fu}-$ sionen und Firmenkäufen diese Zahlen eine sich die Phantasie in vielen Firmen darauf, diese Zahlen möglichst weit in die Höhe zu treiben. Dabei entwickelten Unternehmen neben den auf Marketing basierenden $\mathrm{Me}$ thoden auch technische Möglichkeiten, um die Vorgaben zu erreichen.

Eine Strategie bestand darin, möglichst häufig von Suchrobotern der Internet-Suchmaschinen wie Google oder Altavista aufgesucht zu werden. Diese Suchroboter durchstöbern ununterbrochen das Netz, um ihre Register zu überarbeiten. Dies generiert auf den Websites Eyeballs, Click-Rates und Page Impressions. Da später nicht mehr nachzuprüfen ist, wer eine Website besucht hat, sind die häufigen Besuche durch diese Suchroboter eine Möglichkeit, Zahlen zu schönen. Teilweise verfügten Internetunternehmen auch über kleine Programme, durch die permanent Eyeballs, Click-Rates und Page Impressions auf ihren Seiten generiert wurden. Dies war eine Möglichkeit, Werbekunden positive Zahlen über den Besuch von Werbebannern vorzutäuschen und die für Kapitalgeber so wichtigen Wachstumskriterien zu erfüllen (vgl. Unternehmen E, Marketingleiter).

Je mehr sich jedoch das Wissen um die Manipulationsmöglichkeiten bei ClickRates, Eyeballs und Kundenzahlen verbreitete, desto weniger wurden diese zur Bewertung von Unternehmen herangezogen. Studien, die feststellten, dass häufig drei Viertel aller Klicks auf Werbebanner durch den Betreiber der Website selbst produziert worden waren, um eine hohe Reichweite seiner Seiten vorzugaukeln, führten nicht nur zu einem Vertrauensverlust von Werbekunden in Bezug auf solche Nutzerzahlen, sondern auch die Kapitalgeber nahmen nun Abstand davon, diese Zahlen als maßgebliches Erfolgskriterium zu akzeptieren (vgl. Byrnes 2000: 44; Wippermann et al. 2001: 156; Green/Elgin 2001: 36).

\subsection{Strategien zur Steigerung des Umsatzes}

Der Nachweis von Umsatzsteigerungen war eine weitere zentrale Bewertungskategorie für kapitalmarktorientierte Unternehmen. 
Wenn es gelang, ein rasantes Anwachsen der Einnahmen nachzuweisen, war das Vertrauen von Business Angels, Risikokapitalgebern und Anlegern an den High-TechMärkten groß, dass die hohe Unternehmensbewertung gerechtfertigt war. Eine wesentliche Frage für kapitalmarktorientierte Unternehmen war deshalb, wie man die Umsätze durch eine kreative Buchhaltung besser aussehen lassen konnte.

Eine Strategie, den Umsatz nach oben zu treiben, besteht in Tauschgeschäften mit anderen Unternehmen. Bei solchen BarterGeschäften platzieren Unternehmen beispielsweise auf ihren Websites Werbebanner des jeweils anderen Unternehmens. Die Unternehmen verbuchen die erhaltene Werbung als Einnahme und buchen die beim Konkurrenten geschaltete Werbung als Ausgabe ab. So kann ein Unternehmen mit sehr geringem Aufwand den eigenen Umsatz nach oben treiben, ohne dass ein einziger Dollar oder Euro den Besitzer wechselt. Weil bei einigen Unternehmen diese Tauschgeschäfte mehr als die Hälfte des Unternehmensumsatzes ausmachten, interessierte sich auch die US-amerikanische Börsenaufsicht für diese Praxis.

Firmen, die Produkte im World Wide Web makeln, verbuchen häufig nicht nur die Kommission für die Vermittlung eines Produkts, sondern den Preis für das gesamte Produkt als Einnahme. Maschinen, Flugreisen oder Hotelzimmer werden so behandelt, als ob sie von dem Makler komplett gekauft und dann zu einem höheren Preis an den Endkunden weiterverkauft worden wären. Dies wird auch dann getan, wenn, wie bei Reisebüros, das Geschäft lediglich darin besteht, einen Kunden an eine Fluggesellschaft oder ein Hotel zu vermitteln. Priceline rechnet beispielsweise immer den vollen Preis eines Fluges oder Mietautos als Umsatz ab, obwohl nur ein Bruchteil davon in der Kasse des Unternehmens bleibt.

Eine andere bewährte Strategie zur Steigerung des Umsatzes besteht darin, gewährte Discounts und Rabatte für Computer, Softwareprogramme, Trainings-Dienstleistungen oder Anzeigen gesondert zu verbuchen. Die Einnahmen werden mit dem Komplettpreis verbucht; die gewährten Discounts und Rabatte werden dann als Marketingausgaben verbucht. Der Umsatz des Unternehmens wird so hochgesetzt und die Kosten im von Analysten als nicht so problematisch angesehenen Bereich der Marketingausgaben abgelegt (vgl. Yang 2000: 70; Kühl 2003).

\subsection{Strategien zur Steigerung der Brutto- Handelsspanne}

Von kapitalmarktorientierten Unternehmen wurden in der Wachstumsphase keine Gewinne verlangt, aber sie mussten zunehmend Nachweise dafür liefern, dass sie mit dem Verkauf ihrer Produkte und Dienstleistungen in absehbarer Zeit Gewinn würden machen können. Einem Unternehmen, das für jedes verkaufte Produkt im Wert von zehn Dollar insgesamt 20 Dollar Verlust macht, wird dies von den Kapitalgebern verziehen, wenn die Verluste aufgrund von Marketingausgaben, Investitionen in neue Geschäftsfelder oder Kosten für die internationale Expansion entstehen. Wenn das Unternehmen jedoch bei einem für zehn Dollar verkauften Produkt allein 15 Dollar für die Entgegennahme der Bestellung, das interne Handling in der Firma und die Versandkosten bezahlt, kommen Zweifel am Geschäftsmodell auf.

Die Zahl, die Analysten, Journalisten und Investoren deshalb besonders interessierte, war der „Brutto-Margin“ (gross margin) (vgl. z.B. Sparks 1999: 56). Der Brutto-Margin ist der Anteil an einem eingenommenen Dollar, der einem Unternehmen nach Abzug der Produktions-, Handling- und Zustellkosten bleibt. Er enthält also keine Ausgaben für die Investition in neue Geschäftsfelder oder Marketing. Bei einem Unternehmen wie Microsoft, das einen beeindruckenden Brutto-Margin von 85 Prozent aufweisen kann, heißt dies, dass von einem Dollar, den Microsoft für ein verkauftes Produkt erhält, nur 15 Prozent für die Produktion und die Abwicklung des Versands der Software benötigt wird. Es bleibt also genügend Geld übrig, um Internetunternehmen zu kaufen, Marketingkampagnen zu bezahlen oder sich Rechtsanwälte zu leisten, um Monopolvorwürfe abzuwehren. 
Internetunternehmen, die einen BruttoMargin von über 40 Prozent nachweisen konnten, wurden von Analysten und Journalisten besonders empfohlen, weil ihnen höhere Überlebenschancen in einem möglichen „Online-Preiskrieg" zugetraut wurde. Unternehmen wie eBay oder Homestore wurden deshalb von Beobachtern besonders umhegt, weil sie einen besseren Brutto-Margin aufwiesen als „E-Tailer" wie Amazon, Webvan oder Homegrocer (vgl. Foust 2000: 75). Als zum Beispiel deutlich wurde, dass Amazon bei jeder Bestellung aus verschiedenen Produkten durchschnittlich 2,91 Dollar verlor, weil die Kosten für die Zusammenstellung der Produkte in den Warenlagern und die Versandkosten so extrem hoch waren, kamen Zweifel auf, ob Amazon jemals profitabel werden könnte (vgl. Kerstetter 2000: 45).

Wegen dieses Fokus auf Brutto-Margins versuchten Internetunternehmen, ihre Produktions-, Personal- und Auslieferungskosten als Marketingkosten zu verbuchen und so den Eindruck zu erwecken, dass der Brutto-Margin erfolgversprechend sei. Während traditionelle, überwiegend über Katalog werbende Versandunternehmen Ausführungskosten in der Bilanz unter „Kosten für verkaufte Produkte" verbuchten, versteckten Internetunternehmen wie Drugstore.com und Amazon diese Kosten in ihrem generellen Kostenblock. Einige Servicekosten und Versandkosten wurden als Marketingkosten verbucht und nicht auf den Herstellungspreis angerechnet. So gelang es, ein Bild zu erzeugen, nach dem der Vertrieb einer $C D$, eines Notebook oder einer Beauty-Creme bereits profitabel erschien und das Unternehmen lediglich aufgrund der hohen Marketingkosten noch nicht im schwarzen Bereich lag (Elstrom 2000: 68).

\subsection{Strategien zur Ergebnisverbesserung}

Nachdem Zweifel daran aufgekommen waren, ob die Firmen der New Economy sich durch Werbebanner auf ihren Websites, durch die Bezahlung ihrer Leistungen durch die Kunden oder durch Business-to-Business-Geschäftsmodelle tatsächlich würden finanzieren können, wurde genau darauf geachtet, wie sich die Ergebnisse der Unternehmen entwickelten. In dieser Phase achteten Unternehmen darauf, die Erwartungen von Risikokapitalgebern, Analysten und Medien möglichst zu erfüllen und eine positive Geschäftsentwicklung zu präsentieren.

Eine Strategie, um gute Geschäftsergebnisse vorweisen zu können, bestand darin, die Aufwendungen für die Entwicklung neuer Software oder für Produktforschungen als Vermögen zu verbuchen. Entgegen der nahe liegenden Logik, dass es sich bei solchen Posten um Kosten handelt, die das Ergebnis des Unternehmens mindern, verbuchten einige Unternehmen der New Economy in Übereinstimmung mit der Bilanzierung nach US-GAAP diese Posten als Vermögen. In einer Studie von Karl Heinz Kütting werden verschiedene Beispiele dafür aufgeführt: Der Softwareentwickler Heyde wies beispielsweise allein für 1999 6,9 Millionen an Softwareentwicklungskosten als Vermögen aus und verdoppelte dadurch die Gewinne, die er in seiner Bilanz ausweisen konnte (Fockenbrock/Zdral 2000: 68f).

Eine von New Economy-Unternehmen besonders häufig genutzte Variante war das Hochsetzen der immateriellen Werte wie Patente, Lizenzen und Markenrechte. Karlheinz Kütting stellte fest, dass Firmen der New Economy ihre ,immateriellen Werte“ wie Lizenzen, Patente und Markenrechte in ihren Bilanzen wesentlich höher bewerten als die Unternehmen der Old Economy. Bei Unternehmen wie International Media bestünden $99 \%$ des Anlagevermögens aus immateriellen Werten (vgl. Ogger 2001: 188ff.).

Eine weitere Möglichkeit besteht darin, einen Firmenteil als selbstständiges Tochterunternehmen auszugliedern und die immateriellen Werte des Konzerns dann an dieses Tochterunternehmen zu verkaufen. Die Patente, Markenrechte oder Lizenzen werden an die Tochtergesellschaft veräußert und so das Ergebnis des Konzerns verbessert. Insbesondere durch die rechtliche Ausgliederung von Forschungs- und Entwicklungsabteilungen können so von den Unternehmen Verlustquellen versteckt werden, die sich erst in späteren Jahren in der Konzernbilanz auswirken (vgl. Enzweiler 1999: 107). 


\subsection{Grenzen der Ergebniskosmetik}

Es geht im Folgenden nicht darum zu diskutieren, ob diese - hier nur beispielhaft vorgestellten - Verfahren der Bilanzkosmetik buchhalterisch zu rechtfertigen sind oder nicht. Soziologisch ist interessant, weshalb Firmen der New Economy offenbar in besonders ausgeprägter Weise zu diesen Instrumenten der Bilanzkosmetik griffen, welche Auswirkungen die öffentliche Debatte über die Bilanzierungstricks für die Unternehmen hatte und wie dieser Aspekt mit dem Zusammenbrechen viel versprechender Unternehmen zusammenhing,

Meine These lautet: Die ausgeprägten Formen der Bilanzkosmetik gingen darauf zurück, dass das Geschäftsmodell der meisten New Economy-Unternehmen auf einem permanenten Nachfluss von Geld aus dem Kapitalmarkt aufgebaut waren und deshalb Unternehmen mit aller Gewalt verhindern mussten, Signale auszusenden, die den Kapitalmarkt beunruhigt hätten. Das Ausbleiben von Überweisungen durch die Risikokapitalgeber, die Verminderung der Möglichkeit, über einen Börsengang neues Geld einzusammeln, oder das Verfallen der Unternehmensakquise- und Refinanzierungswährung "Aktie" konnte das schnelle Ende eines kapitalmarktorientierten Unternehmens bedeuten.

Aus dieser Perspektive wird verständlich, weswegen der Kapitalmarkt so sensibel darauf reagierte, wenn starke Entkopplungen, beispielsweise in Form umstrittener Buchungsformen, bekannt wurden und Unternehmen ihre Zahlen korrigieren mussten. Als EM.TV zugeben musste, dass kleinere Fehler bei der Erstellung der Bilanz gemacht worden waren, ging der Kurs an einem Tag um 32 Prozent nach unten. Als man bei Priceline die Zahlen für die verkauften Airline-Tickets nicht mehr künstlich nach oben treiben konnte und die Verkaufserwartungen korrigieren musste, fiel der Kurs an einem Tag um 46 Prozent (vgl. Kerstetter 2000: 45).

Für kapitalmarktorientierte Unternehmen ist das "Management" dieser Entkopplung eine wesentliche Überlebensbedingung. Zugestanden: Die durch offensichtlich werdende Entkopplungen entstehenden Kursverluste sind, wie die Beispiele HypoVereinsbank und Telecom zeigen, auch für primär auf den Verkauf von Produkten ausgerichtete Unternehmen nicht angenehm. Sie stellen aber in der Regel nicht die Überlebensfähigkeit des Unternehmens in Frage. Solange das Unternehmen durch den Verkauf der Produkte und Dienstleistungen mehr einnimmt als es ausgibt, ist seine Liquidität nicht unmittelbar bedroht. Bei kapitalmarktorientierten Unternehmen wirkt sich die Erosion des Vertrauens der Kapitalanleger jedoch häufig verheerend aus. Durch das Ausbleiben von Nachschüssen aus dem Kapitalmarkt wird der Spielraum zur Liquiditätssicherung geringer und mühsam aufgebaute Finanzierungsgerüste drohen zusammenzustürzen.

\section{Schluss: Zusammenhang und Differenz von Produktmarkt- und Kapitalmarktorientierung}

In diesem Artikel wurden zwei unterschiedliche Strategien von Unternehmen zur Aufrechterhaltung ihrer Zahlungsfähigkeit differenziert. Durch die Theorierichtung des NeoInstitutionalismus ist darauf aufmerksam gemacht worden, dass Unternehmen nicht unbedingt operative Gewinne machen müssen, um zu überleben. Staatliche Subventionen, Querfinanzierungen von profitablen zu verlustreichen Unternehmenstöchtern oder Nachschubfinanzierungen über den Kapitalmarkt sind Alternativen zu operativem Gewinn.

Besonders in Boom-Phasen ist die Refinanzierung über den Kapitalmarkt für schnell wachsende Unternehmen attraktiv. Schnelles Wachstum, ein vielversprechendes Produkt und eine überzeugende „Equity Story“ vorausgesetzt, ist das Unternehmensmanagement in der Lage, bei Business Angels, Risikokapitalgebern und Aktionären ausreichend Geld einzusammeln, um die Zahlungsfähigkeit aufrechtzuerhalten.

Was unterscheidet die Strategie der Finanzierung über ,operativen Gewinn" von der über regelmäßige Nachschläge durch den Kapitalmarkt? Wie hängen diese beiden - 
hier idealtypisch unterschiedenen - Finanzierungsstrategien zusammen?

Die Finanzierungsstrategie ,operativer Gewinn", die im Neo-Institutionalismus als eine Fokussierung auf technische Rationalität bezeichnet wird, ist darauf aufgebaut, die Arbeitskraft von Mitarbeitern so einzusetzen, dass die von ihnen produzierten Waren und Dienstleistungen zu einem Preis verkauft werden können, der über den Erbringungskosten liegt. Das ökonomische Kalkül besteht darin, die Kosten für Zulieferprodukte, Maschinen und Arbeitskräfte niedriger $\mathrm{zu}$ halten als die Einnahmen aus dem verkauften Produkt oder der erbrachten Dienstleistung. Solange die Differenz positiv ausfällt, kann das Unternehmen seine Zahlungsfähigkeit aufrechterhalten. Die Differenz zwischen Einnahmen und Kosten, der Gewinn also, kann dann entweder an die Kapitalgeber ausgeschüttet oder für Investitionen genutzt werden.

Die Finanzierungsstrategie über „Nachschüsse aus dem Kapitalmarkt", im NeoInstitutionalismus durch das Konzept der institutionellen Rationalität repräsentiert, basiert darauf, über den Kapitalmarkt immer wieder Gelder einzusammeln, durch die die Zahlungsfähigkeit des Unternehmens auch bei Verlusten im operativen Geschäft aufrechterhalten werden kann. Den Business Angels, Risikokapitalgebern und Aktionären in Wachstumsunternehmen wird dabei das Versprechen gegeben, dass das Unternehmen so schnell wachsen kann, dass der kurzfristige Verzicht auf eine Dividende und die Inkaufnahme eines Investitionsrisikos sich langfristig auszahlen.

Diese auf den Kapitalmarkt ausgerichtete Perspektive zur Aufrechterhaltung der Zahlungsfähigkeit ist insofern mit der ersten Strategie gekoppelt, als ein Versprechen gegeben wird, dass das schnell wachsende Unternehmen nicht nur in absehbarer Zeit die Zahlungsfähigkeit allein durch seine Produkt- oder Dienstleistungsverkäufe wird sicherstellen können, sondern dabei auch so hohe Profite machen wird, dass es eine beachtliche Dividende wird zahlen können. Gib dein Geld - so die Logik - nicht heute auf dem Markt für Gemüse aus, sondern investiere das Geld in Kleingarten, Saat und
Schaufel, dann wirst du langfristig mehr Gemüse dafür bekommen. „Revolution der Wirtschaft", "Lock-in" und "Increasing Returns" sind dabei die Konzepte, die die Hoffnung auf eine beachtliche Dividende in der Zukunft nähren.

Nur weil es einen ,time gap “ zwischen der Investitionsphase, in der das Unternehmen nicht profitabel ist, und der Profitabilitätsphase gibt, kann überhaupt ein Geschäft mit Risikokapital entstehen. Business Angels, RisikokapitalgeselIschaften und Anleger in Aktien von Wachstumsunternehmen können nur deswegen mit höheren Renditen rechnen, weil sie das Risiko eingehen, dass ein Unternehmen diesen Übergang nicht schafft und liquidiert werden muss. Egal ob man dies als "Handel mit Phantasie“, als "Spekulation“ oder als „Wette auf die Zukunft" bezeichnet - wenn es das Risiko nicht gäbe, wäre es für einen Unternehmer nicht sinnvoll, zu verhältnismäßig schlechten Konditionen Teile seines Eigenkapitals abzugeben. Bei einer ,sicheren" Investition in die Zukunft wäre es einfacher und günstiger, einen Kredit bei einer Bank aufzunehmen.

Das Besondere am Risikokapitalmarkt ist, dass die Business Angels, Risikokapitalgeber und Anleger nicht warten müssen, bis das finanzierte Unternehmen in eine Phase der Profitabilität eingetreten ist und Dividenden an die Kapitalanleger auszahlt. Die Anteile an einem Wachstumsunternehmen sind selbst handelbar. Damit besteht die Möglichkeit für Anteilseigner, aus einem Unternehmen auszusteigen, bevor Rentabilität erreicht ist. Bedingung ist lediglich, dass andere Anleger die Zukunft des Unternehmens als so gut einschätzen, dass sie bereit sind, einen Preis für die Anteile zu bezahlen, der über den ursprünglichen Investitionen des Risikokapitalgebers liegt.

Nun verlagern sich die Perspektiven immer mehr vom Produktmarkt auf den Kapitalmarkt. Zwar sind immer wieder Verweise darauf nötig, dass sich die Produkte in näherer Zukunft werden verkaufen lassen, im Mittelpunkt des Interesses steht jedoch die Refinanzierung über den Kapitalmarkt. Es entstehen primär kapitalmarktorientierte Unternehmen, deren Expansionsstrategien, Marketingkampagnen und Buchhaltungs- 
weisen auf die Sicherung des Nachschubs der Kapitalmarktfinanzierung ausgerichtet sind. Dabei spielt die Bezugnahme auf Mythen eine zentrale Rolle.

Der Neo-Institutionalismus hat darauf aufmerksam gemacht, dass Mythen immer dann eine prominente Rolle spielen, wenn Entscheidungen durch sehr hohe Unsicherheit belastet sind. Im Fall der Risikokapitalfinanzierung ist die starke Orientierung an der Zukunft (z.B. „Profitabilität in drei Jahren“, „potenzieller Weltmarktführer", "langfristiges Wachstumspotenzial") das Einfallstor für Mythen. Weil Aussagen über die Zukunft immer mehr oder minder unsichere Aussagen sind, besteht im Fall der Risikokapitalfinanzierung eine starke Tendenz, als Erfolgsmodell gehandelte $\mathrm{Ge}$ schäftsmodelle zu kopieren und sich an den allgemein geteilten Mythen über zukünftige Wirtschaftsentwicklungen zu orientieren.

\section{Anmerkungen}

1 Der Artikel basiert vorrangig auf einer Auswertung von Interviews und Artikeln über die New Economy in deutschen, britischen und US-amerikanischen Zeitschriften sowie von Büchern über die New Economy. Diese dienen mir zur Rekonstruktion des öffentlichen Diskurses von Unternehmern, Risikokapitalgebern, Analysten und Journalisten. Die Analyse der Interviews, Artikel und Bücher wird ergänzt durch qualitative Interviews in fünf Firmen der New Economy und Expertengespräche mit Geschäftsführern und Mitarbeitern von neun Risikokapitalgesellschaften. Zur Sicherstellung der Anonymität der Unternehmen und der Mitarbeiter wurden Angaben, die für die Argumentation nicht zentral sind (Produkt, Zeiten, Financiers, Zuordnung von Gesprächspartnern), verändert. Die Ergebnisse aus den Interviews wurden insbesondere zum Abgleich mit den veröffentlichten Quellen genutzt und dienen der Validierung der öffentlich zugänglichen Quellen. In dem Abschnitt, in dem es um die Strategien zur Erreichung von Meilensteinen geht, greife ich vorrangig auf Arbeiten aus der Betriebswirtschaftslehre zurück, in denen die Buchhaltungsmethoden von börsennotierten Unternehmen unter die Lupe genommen werden.

\section{Literatur}

Arthur, W. Brian (1994): Increasing Returns and Path Dependence in the Economy. Ann Arbor: University of Michigan Press.

Brady, Diane (1999): Jay S. Walker. In: Business Week E.biz, 27.9.1999, S. 22.

Brunsson, Nils (1985): The Irrational Organization. Irrationality as a Basis for Organizational Action and Change. Chichester: John Wiley.

Bymes, Nanette (2000): Eyeballs, Bah! Figuring Dot-Coms' Real Worth. In: Business Week, 30.10.2000, S. 44.

Capell, Kerry (2000): Britain.com. In: Business Week, 6.3.2000, S. 22-26.

Castells, Manuel (2000): The Rise of the Network Society. 2. überarb. Aufl. Cambridge/Oxford: Blackwell.

Cronin, Mary J. (2000): Unchained Value. The New Logic of Digital Business. Boston: Harvard Business School Press.

Davis, Stan/Christopher Meyer (1998): Blur. The Speed of Change in the Connected Economy. Reading, Mass.: Addison-Wesley.

DiMaggio, Paul J./Walter W. Powell (1991): Introduction. In: Walter W. Powell/Paul J. DiMaggio (Hrsg.), The New Institutionalism in Organizational Analysis. Chicago/London: University of Chicago Press, S. 1-38.

Echikson, William (2000): A Blip - or a Bad Omen. In: Business Week, 3.4.2000, S. 22-25.

Elstrom, Peter (2000): The End of Fuzzy Math? In: Business Week E.Biz, 11.12 .2000 , S. 6869.

Enzweiler, Tasso (1999): Die Bilanzjongleure. Wie deutsche Unternehmen ihre Aktionäre und die Öffentlichkeit in die Irre führen. Frankfurt a.M.: Societäts-Verlag.

Evans, Philip/Thomas S. Wurster (2000): Blown to Bits: How the New Economics of Information Transforms Strategy. Boston: Harvard Business School Press.

Farrell, Christopher (1999): All the World's an Auction Now. In: Business Week, 4.10.1999, S. 70-74.

Feng, Hengyi et al. (2001): A New Business Model? Berlin: Discussion Papers WZB FS II 01-202.

Fockenbrock, Dieter/Wolfgang Zdral (2000): Die Tricks der Finanz-Jongleure. In: Capital 20, S. 66-89.

Foust, Dean (1999): Time to Buy Net Stocks? No ... In: Business Week, 17.5.1999, S. 58-59.

Foust, Dean (2000): Some Dot-Com Jewels Will Shine Again. In: Business Week, 25.12.2000, S. 74-75. 
France, Mike (1999): A Net Monopoly No Longer? In: Business Week, 27.9.1999, S. 90.

Froitzheim, Ulf (2001): New Economy von A bis Z. In Bizz 1, Sonderbeilage Bizz-Guide, S. 330.

Green, Heather (1999): Mary G. Meeker. In: Business Week E.Biz, 2.9.1999, S. 35.

Green, Heather/Ben Elgin (2001): Do e-Ads Have a Future? In: Business Week E.Biz, 22.1.2001, S. 36-40.

Hagel III, John/Arthur G. Armstrong (1997): Net Gain. Expanding Markets Through Virtual Communities. Boston: Harvard Business School Press.

Harmon, Steve (2001): Aufstieg und Erfolg im Internet. Wenn aus Visionen Werte werden. München: FinanzBuch.

Hartman, Amir/John Sifonis (2000): Net Ready. Strategies for Success in the E-conomy. New York: McGraw-Hill.

Himelstein, Linda (1999): Yes ... In: Business Week, 17.5.1999, S. 59.

Hof, Robert D. (2000): E-Marketplaces. A Bloodbath. In: Business Week E.Biz, 3.4.2000, S. 72 .

Hof, Robert D. (2001): Remember the Tortoise. In: Business Week E.Biz, 22.1.2001, S. 50.

Huchler, Norbert (2002): Rationalitätsmythen der New Economy. München: www.dotcom-research de.

Kelly, Kevin (1998): New Rules for the New Economy. New York: Viking Pinguin.

Kerstetter, Jim (2000): The Dark Side of the Valley. In: Business Week, 17.7.2000, S. 4445.

Krafft, Lutz (2002): Überlebenschance 10 Prozent. In: changeX.de/d_a00483print.html.

Kühl, Stefan (2003): Exit. Wie Risikokapital die Regeln der Wirtschaft verändert. Frankfurt a.M./New York: Campus.

Laderman, Jeffrey M. (1999): A Turbocharge from Tech. In: Business Week, 27.12.1999, S. 52-53.

Madrick, Jeff (1999): How New is the New Economy? In: The New York Times Review of Books, 23.9.1999.

Malone, Michael (1997): New Metrics for a New Age. In: Forbes, Jg. 159, Heft 7, S. 40-43.

Mandel, Michael J. (1999): The Internet Economy: The World's Next Growth Engine. In: Business Week, 4.10.1999, S. 44-49.
Mandel, Michael J. (2000): crash.com. München: Financial Times Prentice Hall.

Meyer, John W./Brian Rowan (1977): Institutionalized Organizations. Formal Structure as Myth and Ceremony. In: American Journal of Sociology 83, S. 340-363.

Meyer, Marshall W./Lynne Zucker (1989): Permanently Failing Organizations. London: Sage.

Modahl, Mary (2000): Now or Never. How Companies Must Change Today to Win the Battle for Internet Consumers. New York: HarperBusiness.

Ogger, Günter (2001): Der Börsenschwindel. Wie Aktionäre und Anleger für dumm verkauft werden. München: C. Bertelsmann Verlag.

Rifkin, Jeremy (2000): Access. Das Verschwinden des Eigentums. Frankfurt a.M./New York: Campus.

Shiller, Robert J. (2000): Irrational Exuberance. Princeton: Princeton University Press.

Southwick, Karen (2001): The Kingmakers. Venture Capital and the Money behind the Net. New York: John Wiley \& Sons.

Sparks, Debra (1999): Who Will Survive the Internet Wars? In: Business Week, 27.12.1999.

Tapscott, Don (1996): The Digital Economy. Promise and Peril in the Age of Networked Intelligence. New York: McGraw-Hill.

Tapscott, Don/David Ticoll/Alex Lowy (2000): Digital Capital: Harnessing the Power of Business Web. Boston: Harvard Business School Press.

Walgenbach, Peter (2000): Die normgerechte Organisation. Eine Studie über die Entstehung, Verbreitung und Nutzung der DIN EN ISO $9000 \mathrm{er}$ Normreihe. Stuttgart: SchäfferPoeschel.

Walgenbach, Peter (2002): Neoinstitutionalistische Organisationstheorie - State of the Art und Entwicklungslinien. In: Georg Schreyögg/ Peter Conrad (Hrsg.), Managementforschung 12. Wiesbaden: Gabler Verlag, S. 155-202.

Winblad, Ann (2001): Vorwort. In: Steve Harmons (Hrsg.), Aufstieg und Erfolg im Internet. Wenn aus Visionen Werte werden. München: FinanzBuch, S. 5-9.

Wippermann, Peter et al. (2001): Wörterbuch der New Economy. Mannheim et al.: Dudenverlag.

Yang, Catherine (2000): Earth to Dot-Com Accountants. In: Business Week, 3.4.2000, S. 70. 